\title{
Universiteit
}

Leiden

The Netherlands

\section{Role of dissimilar interfaces in thin films of cylinder-forming block copolymers}

Lyakhova, K.S.; Sevink, G.J.A.; Zvelindovsky, A.V.; Horvat, A.; Magerle, R.

\section{Citation}

Lyakhova, K. S., Sevink, G. J. A., Zvelindovsky, A. V., Horvat, A., \& Magerle, R. (2004). Role of dissimilar interfaces in thin films of cylinder-forming block copolymers. Journal Of Chemical Physics, 120(2), 1127-1137. doi:10.1063/1.1632475

Version: $\quad$ Not Applicable (or Unknown)

License: $\quad$ Leiden University Non-exclusive license

Downloaded from: https://hdl.handle.net/1887/64328

Note: To cite this publication please use the final published version (if applicable). 


\section{Role of dissimilar interfaces in thin films of cylinder-forming block copolymers}

K. S. Lyakhova, G. J. A. Sevink, A. V. Zvelindovsky, A. Horvat, and R. Magerle

Citation: The Journal of Chemical Physics 120, 1127 (2004); doi: 10.1063/1.1632475

View online: https://doi.org/10.1063/1.1632475

View Table of Contents: http://aip.scitation.org/toc/jcp/120/2

Published by the American Institute of Physics

\section{Articles you may be interested in}

Phase behavior in thin films of cylinder-forming ABA block copolymers: Experiments

The Journal of Chemical Physics 120, 1105 (2004); 10.1063/1.1627324

Thin films of block copolymer

The Journal of Chemical Physics 106, 7781 (1997); 10.1063/1.473778

Asymmetric block copolymers confined in a thin film

The Journal of Chemical Physics 112, 2452 (2000); 10.1063/1.480811

Phase behavior in thin films of cylinder-forming ABA block copolymers: Mesoscale modeling

The Journal of Chemical Physics 120, 1117 (2004); 10.1063/1.1627325

Block Copolymers-Designer Soft Materials

Physics Today 52, 32 (1999); 10.1063/1.882522

Fluctuation effects in the theory of microphase separation in block copolymers

The Journal of Chemical Physics 87, 697 (1987); 10.1063/1.453566

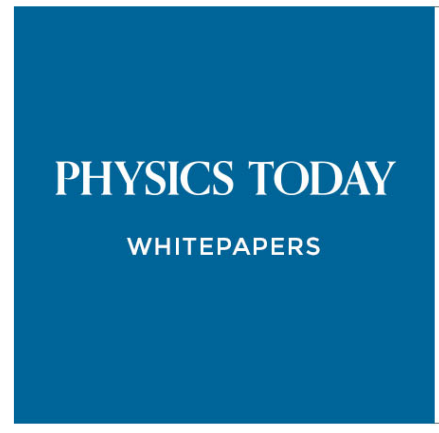




\title{
Role of dissimilar interfaces in thin films of cylinder-forming block copolymers
}

\author{
K. S. Lyakhova, G. J. A. Sevink, ${ }^{a)}$ and A. V. Zvelindovsky \\ Leiden Institute of Chemistry, Gorlaeus Laboratory, Leiden University, P.O. Box 9502, \\ 2300 RA Leiden, The Netherlands
}

\author{
A. Horvat and R. Magerle \\ Physikalische Chemie II, Universitaet Bayreuth, D-95440 Bayreuth, Germany
}

(Received 19 August 2003; accepted 16 October 2003)

\begin{abstract}
We study the effect of dissimilar interfaces on the phase behavior of cylinder forming block copolymers in thin films by means of dynamic density-functional theory. In this article, we show that dissimilarity of the interfaces induces hybrid structures. These structures appear when the surface fields at the two interfaces stabilize different surface structures and/or reconstructions. We propose a general classification of hybrid structures and give an unifying description of phase behavior of cylinder forming block copolymer films. Our results are consistent with experimental observations. (C) 2004 American Institute of Physics. [DOI: 10.1063/1.1632475]
\end{abstract}

\section{INTRODUCTION}

It is well-known that block copolymers self-assemble into mesostructures at certain temperatures. These mesostructures are receiving increasing attention, ${ }^{1,2}$ since selforganization can be utilized in various applications in nanotechnology. Shearing, electric fields, temperature gradients, and confinement are frequently employed to induce long range ordering of structure resulting from spontaneous selfassembly. Understanding and controlling the important factors of structures formation of these materials is therefore of great interest.

Numerous studies have dealt with thin films of block copolymers that form lamellar structures in bulk (for an overview see Refs. 2-4). Two major factors have been identified to control structure formation: (1) The preferential attraction of one type of block to the surface (the surface field) causes the lamella to align parallel to the interfaces and (2) the system's natural microdomain spacing causes the film to form islands or holes (terraces) where the film thickness is a half integer multiple of bulk lamella spacing.

In systems that form other microdomain structures in bulk, such as cylinders, the situation is more complex and much less studied. While any cross section parallel to a lamella exhibits the same symmetry as a planar surface, this is not the case in cylinder forming systems. Here, a planar surface, regardless of its orientation, always breaks the symmetry of the bulk structure and the microdomain structure has to adjust. For cylinder forming systems, a variety of deviations from the bulk structure have been observed near surfaces and in thin films such as a wetting layer, ${ }^{5}$ spherical microdomains, ${ }^{6}$ a perforated lamella, ${ }^{6}$ cylinder with necks, ${ }^{7}$ and more complicated structures. ${ }^{8}$ The phase behavior for cylinder forming systems was studied in detail for diblock, ${ }^{9-15}$ and for triblock copolymers. ${ }^{16-19}$

\footnotetext{
a) Author to whom correspondence should be addressed. Electronic mail: a.sevink@chem.leidenuniv.nl
}

In previous work, ${ }^{16,17}$ we have simulated thin films with similar interfaces, where the film-interface interactions are equal at both interfaces. Based on a comparison of experiments and simulations for a polystyrene-block-polybutadienblock-polystyrene (SBS) triblock copolymer, we have shown ${ }^{16,17}$ that the phase behavior in this type of films is dominated by surface reconstructions. ${ }^{20}$ While in the case of lamella forming diblock copolymers ${ }^{3}$ only the orientation of the lamella depends on the two parameters mentioned above, for cylinder forming systems also the type of microdomain structure can change. The stability regions of the different surface structures and surface reconstructions are determined by the surface field and the film thickness, and we have shown how these two effects interact.

In experiments, supported films are usually studied that are asymmetric with respect to interactions at the air-film and film-substrate interfaces. In addition, several types of di- and triblock copolymers are employed as compatibilizers in binary and ternary blends to enhance the macroscopic material properties. ${ }^{21-23}$ As these copolymers usually migrate to the blends interfaces, this gives rise to a confined situation, and the observed triblock structures at the blend interfaces might be compared to our findings. One problem is that this situation is much more complex than the case of supported films: Apart from the asymmetry in the (unknown) interface interactions, both interfaces themselves are deformable. Besides complex, the study is far from complete and scattered over many types of block copolymers. We, therefore, focus our comparison to the experimentally observed phase behavior in supported films. ${ }^{24}$ In a previous article we parametrized this system and showed that its behavior can be well described by simulations with symmetric boundaries. ${ }^{16}$ In the present article, we would like to understand the reasons for this paradox behavior and study in detail the role of dissimilar interfaces on the phase behavior in thin films of cylinder forming block copolymers.

In theoretical studies ${ }^{25-28}$ dissimilar interfaces were 


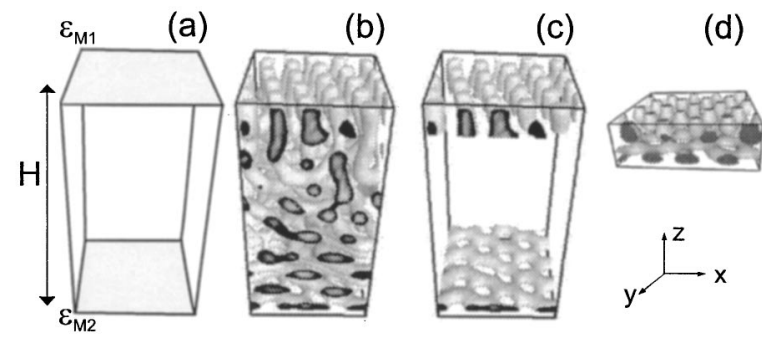

FIG. 1. (a) The geometry of the simulation box. (b) The effect of dissimilar interfaces on the microdomain structures of an $A_{3} B_{12} A_{3}$ block copolymer melt in a film of thickness $H=10 c_{0}$ and different interaction parameters $\varepsilon_{M_{1}}$ and $\varepsilon_{M_{2}}$ at the two interfaces. At the top, cylinders orient perpendicular to the interface, at the bottom, a perforated lamella is stabilized. In the middle of the film, cylinders form corresponding to the bulk structure of this system (Ref. 17). (c) Same as in (b) but only layers next to the interface are shown. (d) The same as in (b) for $H=1.5 c_{0}$.

mostly studied for systems that form lamellae in bulk. In cases where the two confining interfaces favor different orientations of lamella $\left(\mathrm{L}_{\|}, \mathrm{L}_{\perp}\right)$, these two orientations can coexist and a hybrid structure (HY) forms. ${ }^{28}$ This is also observed in experiments. ${ }^{28}$ In thin films of cylinder forming block copolymers, cylinders with necks have been observed, ${ }^{7}$ which are a combination of parallel and perpendicular oriented cylinders. This is one example of a so-called hybrid structure. Another experimentally observed hybrid structure is a layer of spheres on top of a perforated lamella. ${ }^{29}$ The interplay between the strength of the two surface fields, their dissimilarity, and the film thickness is expected to cause a complex phase behavior, an example of which is shown in Fig. 1.

In the following, we analyze the phase behavior as a function of the film thickness $H$ and the two surface fields. In addition to the phases already observed in the symmetric case, ${ }^{16}$ we find a rich zoo of hybrid structures. We set up an unifying classification for these microdomain structures and explain the physics behind this complex phase behavior.

\section{MODEL}

We model the polymer film as a collection of Gaussian chains $A_{N_{A} / 2} B_{N_{B}} A_{N_{A} / 2}$, with a total length $N=N_{A}+N_{B}$, each representing a triblock copolymer molecule in a mean field environment. The polymer is confined between two parallel solid surfaces. Dynamic density-functional theory (DDFT) $)^{19,30-32}$ is used to describe the temporal evolution of the system. We describe the simulation procedure only briefly. ${ }^{33,34}$ The free energy functional has the form

$$
\begin{aligned}
F[\rho]= & -k T \ln \frac{\Phi^{n}}{n !}-\sum_{I} \int_{V} U_{I}(\mathbf{r}) \rho_{I}(\mathbf{r}) d \mathbf{r} \\
& +\frac{1}{2} \sum_{I, J} \int_{V^{2}} \varepsilon_{I J}\left(\left|\mathbf{r}-\mathbf{r}^{\prime}\right|\right) \rho_{I}(\mathbf{r}) \rho_{J}\left(\mathbf{r}^{\prime}\right) d \mathbf{r} d \mathbf{r}^{\prime} \\
& +\frac{1}{2} \sum_{\alpha} \sum_{I} \int_{V^{2}} \varepsilon_{I M_{\alpha}}\left(\left|\mathbf{r}-\mathbf{r}^{\prime}\right|\right) \rho_{I}(\mathbf{r}) \rho_{M_{\alpha}}\left(\mathbf{r}^{\prime}\right) d \mathbf{r} d \mathbf{r}^{\prime} \\
& +\frac{\kappa_{H}}{2} \int_{V}\left(\sum_{I} \nu\left(\rho_{I}(\mathbf{r})-\rho_{I}^{0}\right)\right)^{2} d \mathbf{r},
\end{aligned}
$$

where $n$ is the number of polymer molecules, $\Phi$ is the intramolecular partition function for ideal Gaussian chains, $\rho_{I}$ is the density of the copolymer component $I$ (in our case $A$ and $B$ ), and $V$ is the system volume. The external potentials $U_{I}$ are conjugate to the densities $\rho_{I}$ via the Gaussian chain density functional. ${ }^{30}$ The volumes of all bead types are chosen to be equal $\left(\nu_{A}=\nu_{B}=\nu\right)$. The forth term models the asymmetric interaction between polymer beads and the top and bottom interface. The different interfaces, which are only different in terms of interaction $\varepsilon_{I M_{\alpha}}(\mathbf{r})$ and location, are labeled by an index $\alpha$. The interface-bead interaction and beadbead interaction have the same Gaussian kernel

$$
\varepsilon_{I J}\left(\left|\mathbf{r}-\mathbf{r}^{\prime}\right|\right)=\varepsilon_{I J}^{0}\left(\frac{3}{2 \pi a^{2}}\right)^{3 / 2} e^{-3 / 2 a^{2}\left(\mathbf{r}-\mathbf{r}^{\prime}\right)^{2}}
$$

where $a$ represents the bond length. The surface field is of relatively short range. However, the system experiences this interaction away from the surfaces due to the connectivity of the beads. In spirit of our previous work the extrema of the free energy functional $F$ are found in a dynamic fashion. The time evolution of the density fields is described by the time dependent Landau-Ginzburg-type of equation (see, for instance, Refs. 30, 32, and 34)

$$
\frac{\partial \rho_{I}}{\partial t}=M_{I} \nabla \cdot \rho_{I} \nabla \mu_{I}+\eta_{I},
$$

where $M_{I}$ is the mobility of the different components of the chain, $\mu_{I}$ are the chemical potentials with proper boundary conditions at the surfaces ${ }^{34}$ and $\eta_{I}$ is a noise field that satisfies the fluctuation-dissipation theorem.

In the model presented here, the film is confined between two solid interfaces. In most experiments, however, films are placed on a solid substrate; the other side is a deformable interface (solvent, solvent vapor, or air). In these films, terrace formation is an important phenomena, since in this way unfavorable film thickness can be avoided by the system. Some information about the formation of terraces can be deduced from films confined between two hard surfaces by analyzing the free energy as a function of the film thickness $H .{ }^{9,25}$ We will not consider this approach in the current article.

\section{PARAMETERIZATION}

The system studied in this article is identical to the system of Refs. 16 and 17, including all simulation parameters, except for the dissimilar interface-bead interactions. It consists of a melt of $A_{3} B_{12} A_{3}$ Gaussian chain molecules with equal bead mobilities $M_{A}=M_{B}=M$. The dimensionless time step was set to $\Delta \tau=M \Delta t / h^{2} k T=0.73 .{ }^{9}$ In the vicinity of surfaces rigid wall boundary conditions are used, ${ }^{34}$ and periodic boundary conditions in all Cartesian directions where no walls are present. The $A-B$ interaction $\varepsilon_{A B}^{0}=6.5$ was chosen such that the experimental and simulated phase diagrams match best, for details see Refs. 16 and 17. With this interaction the distance between next nearest cylinders in the bulk was found to be $a_{0}=7 \pm 0.5$ grid cells. The resulting distance between subsequent horizontal layers of cylinders is, therefore, $c_{0}=(\sqrt{3} / 2) a_{0} \approx 6$ grid cells. 
All simulations were started from the same initial condition, corresponding to uniform $A-B$ density distributions (complete mixing) with external fields $U_{I}=0$. The integration procedure was carried out as long as the density fields change significantly by monitoring the phase separation process with the help of an order parameter $P .{ }^{30}$ The average simulation time was 4000 dimensionless timesteps, however, we checked the stability of some nonperfect structures by continuing simulations till 8000 or more time steps. All simulations were performed in a box of size $32 \times 32$ $\times(H+2)$. The two substrates $M_{1}$ and $M_{2}$ are located at $z$ $=1$ and $z=H+2$, respectively, and span the box in the $x$ and $y$ direction completely. We have four different surface interaction parameters $\varepsilon_{A M_{1}}^{0}, \varepsilon_{A M_{2}}^{0}, \varepsilon_{B M_{1}}^{0}$, and $\varepsilon_{B M_{2}}^{0}$. Since for each surface only the difference between interaction parameters counts in the chemical potential, we introduce effective interactions $^{19} \quad \varepsilon_{M_{1}}=\left(\varepsilon_{A M_{1}}^{0}-\varepsilon_{B M_{1}}^{0}\right) / \nu k T$ and $\varepsilon_{M_{2}}=\left(\varepsilon_{A M_{2}}^{0}\right.$ $\left.-\varepsilon_{B M_{2}}^{0}\right) / \nu k T$, which reduces the number of surface interaction parameters to two. We have explored the parameter space $\varepsilon_{M_{1}}, \varepsilon_{M_{2}}, H$ by integer increments. The resulting density fields $\rho_{A}(\mathbf{r})$ were visualized by an isosurface with threshold value 0.5 and classified by visual inspection.

\section{RESULTS AND DISCUSSION}

\section{A. Three-dimensional (3D) phase diagram}

In this article we consider four planes in the threedimensional (3D) diagram (Fig. 2), with the film thickness $H$ (in grid-cells) on the vertical axis and the effective surface interactions $\varepsilon_{M_{1}}$ and $\varepsilon_{M_{2}}$ (in $\mathrm{kJ} / \mathrm{mol}$ ) on the horizontal axis. In Fig. 2(b) the location of the planes $H=c_{0}, H=3 / 2 c_{0}$, $H=2 c_{0}$ and the plane $\varepsilon_{M_{1}}=6$ are depicted. The results depicted in detail for $\varepsilon_{M_{1}}=\varepsilon_{M_{2}}$ were previously published in Ref. 16.

Surface structures of parallel $\left(\mathrm{C}_{\|}\right)$and perpendicular $\left(\mathrm{C}_{\perp}\right)$ oriented cylinders as well as the surface reconstructions perforated lamella (PL), lamella (L), and wetting layer (W) were already observed in Ref. 16. An overview of these basic surface structures is shown in Fig. 3. For the description of hybrid structures we introduce the following notation and classification scheme:

(i) Two $A$-rich layers separated by a $B$-rich layer are denoted by a hyphen $(-)$ between the corresponding $A$ structures, for example, $\mathrm{PL}-\mathrm{C}_{\perp}$ in Fig. 1(d);

(ii) lateral coexistence of $A$-structures in one horizontal layer is denoted by a slash (/), for example, $\mathrm{C}_{\|} / \mathrm{PL}$ in Fig. 10 in Horvat et al. $;^{17}$

(iii) no separating symbol is used for connected structures, for example, $\mathrm{C}_{\|} \mathrm{C}_{\perp}$, which denotes cylinders with necks. $^{7}$

\section{The vertical plane $\left(\varepsilon_{M 1}=6, \varepsilon_{M 2}, H\right)$}

First we consider the phase diagram for constant $\varepsilon_{M_{1}}$ $=6$ varying $H$ and $\varepsilon_{M_{2}}$. Since the phase diagram is invariant with respect to the interchange of $\varepsilon_{M_{1}}$ by $\varepsilon_{M_{2}}$, this slice represents two pairwise orthogonal slices in the phase diagram (Fig. 2) at $\varepsilon_{M_{1}}=6$ and $\varepsilon_{M_{2}}=6$.
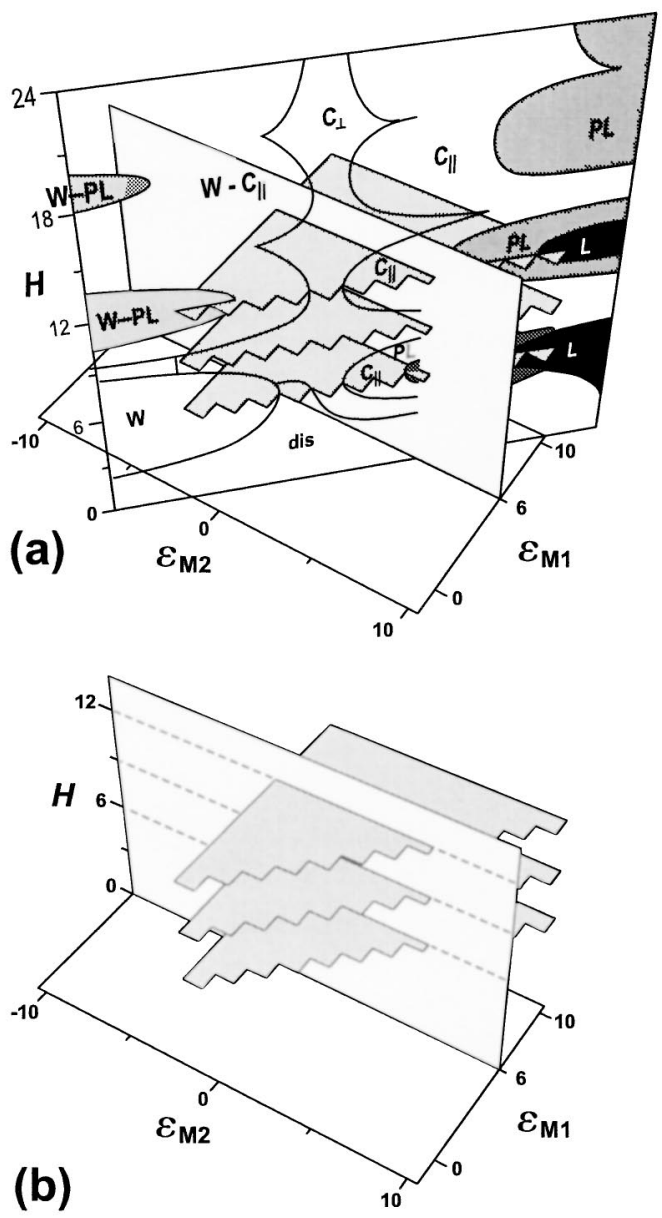

FIG. 2. (a) Schematic 3D phase diagram of surface structures in thin films of $A_{3} B_{12} A_{3}$ block copolymers as a function of the film thickness $H$ and two effective interactions of the polymer with the surfaces $\varepsilon_{M_{1}}$ and $\varepsilon_{M_{2}}$. The interaction $\varepsilon_{M_{1}}=\varepsilon_{M_{2}}$ corresponds to the symmetric case published in Ref. 16. (b) Subset of (a) representing the parameter space covered in this article. (Constant film thickness $H=c_{0}, H=3 / 2 c_{0}, H=2 c_{0}$, and constant interaction with one of the surfaces $\varepsilon_{M_{1}}=6$.)

In Fig. 4 the $2 \mathrm{D}$ phase diagram is shown in the range $-10<\varepsilon_{M_{2}}<10(\Delta \varepsilon=1)$ and $3<H<14(\Delta H=1)$. The microdomain structures are shown for $\varepsilon_{M_{2}}=6$ (equal interface-bead interactions) and for $\varepsilon_{M_{2}}=-3$ (dissimilar interface-bead interactions). In the latter case, due to preferential

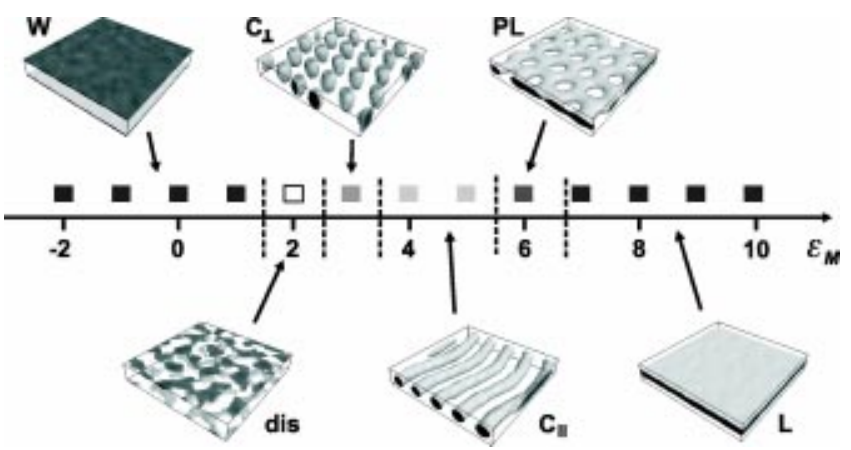

FIG. 3. The basic surface structures for a cylinder forming block copolymer $A_{3} B_{12} A_{3}$ as a function of the surface fields $\varepsilon_{M}=\varepsilon_{M_{1}}=\varepsilon_{M_{2}}$ (from Ref. 17). 


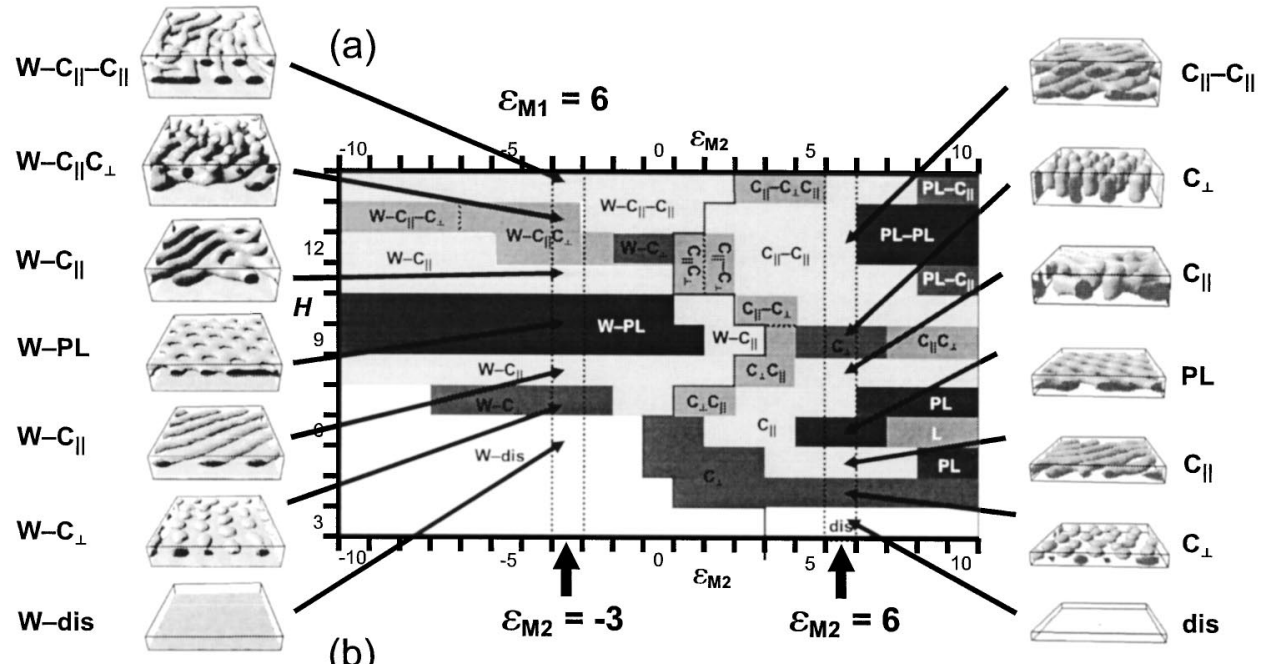

(b)

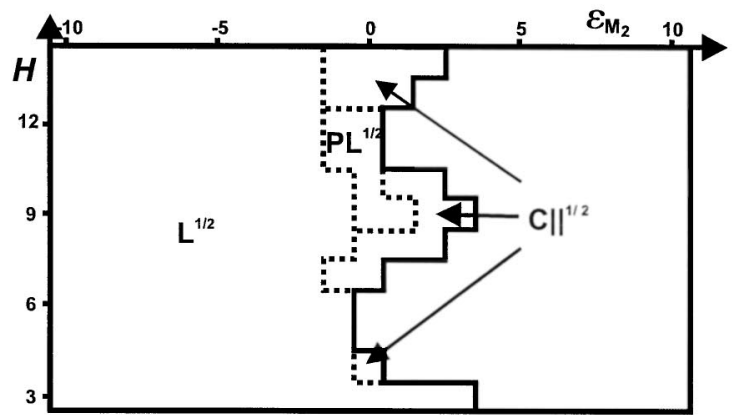

FIG. 4. (a) The phase diagram as a function of the film thickness $H$ and effective surface interaction $\varepsilon_{M_{2}}$ at constant $\varepsilon_{M_{1}}=6$. The microdomain structures that correspond to equal $\left(\varepsilon_{M_{2}}=6\right)$ and to dissimilar $\left(\varepsilon_{M_{2}}\right.$ $=-3$ ) surface interactions are shown. For each square, the structure found at its center point $\left.\varepsilon_{M_{1}}, H\right\}$, was determined by visual inspection and colored by different shades of gray. The border of the region where a wetting layer forms is indicated by a solid line. (b) Structure of the wetting layer in (a). The homogeneous wetting layer corresponds to a half lamella, which is denoted as $\mathrm{L}^{1 / 2}$. Accordingly, structured wetting layers can be considered as half structures denoted by $\mathrm{PL}^{1 / 2}$, $\mathrm{C}_{\|}^{1 / 2}$, and $\mathrm{C}_{\perp}^{1 / 2}$. attraction of $A$ blocks to one of the interfaces, a wetting layer is formed at this interface.

All microdomain structures observed in Fig. 4 are combinations of basic surface structures shown in Fig. 3. A distinct feature is that for $\varepsilon_{M_{2}} \leqslant 2$ a wetting layer forms at one side of the film. The border of this region depends on the film thickness $H$. The phase diagram can roughly be divided into three regions. For $\varepsilon_{M_{2}} \leqslant-1$ the wetting layer is homogeneous and has no lateral structure. In this region the microdomain structures are almost independent of $\varepsilon_{M_{2}}$. For $\varepsilon_{M_{2}} \geqslant 4$ the microdomain structures are mostly basic surface structures and the phase behavior is similar to that for equal interfaces. ${ }^{16,17}$ Between these two regions $\left(-1<\varepsilon_{M_{2}}<4\right)$ different hybrid structures form and the type of the structure is very sensitive to the film thickness and the combination of interaction parameters $\varepsilon_{M_{1}}$ and $\varepsilon_{M_{2}}$. The wetting layer formed in this regions is structured and is complementary to the microdomain structure of its neighboring layer [Fig. 4(b)]. For a detailed discussion of this effect see Ref. 17. We have studied the thickness of the wetting layer by plotting the laterally averaged density profiles $\left\langle\rho_{A}\right\rangle_{x, y}$ as a function of $z$. The effective thickness of the wetting layer was found to be $c_{0} / 2$ regardless whether the wetting layer is structured or not.

The phase behavior in the region $\varepsilon_{M_{2}} \leqslant-1$ can be explained in the following way. When a wetting layer is present, it effectively reduces the film thickness and screens the surface field, in the sense discussed in Huinink et al. ${ }^{10}$ Following this idea, for a homogeneous wetting layer, the effective surface interaction at the side of the wetting layer is
$\varepsilon_{A B}$, rather than $\varepsilon_{M_{1}}$ or $\varepsilon_{M_{2}}$. Indeed we observe that in this region at a certain film thickness $H$ the same microdomain structures forms above the wetting layer as for $\varepsilon_{M_{2}}=6$ $\approx \varepsilon_{A B}$ and $H-c_{0} / 2$.

In the other regions each interface creates its own surface structure depending on the surface field acting at each interface. The two are rather independent of each other as long as the film thickness is large enough. In thinner films the two surface fields add ${ }^{16}$ and the microdomain structure depends on how the two surface structures fit and in some cases connect to each other. The details of this are determined by the commensurability effects both in the $z$ and in the $(x, y)$ direction.

Next we consider three horizontal planes $H=c_{0}, H$ $=3 / 2 c_{0}$, and $H=2 c_{0}$ of the $3 \mathrm{D}$ phase diagram [Fig. 2(b)] in order to separate two effects: the interplay between the confinement and deformability of structures and the additivity of surface fields. For all planes the effective interactions $\varepsilon_{M_{1}}$ and $\varepsilon_{M_{2}}$ are chosen in the range $[-1,10]$ with integer increment. The three diagrams are symmetric with respect to the diagonal $\varepsilon_{M_{1}}=\varepsilon_{M_{2}}$ (bottom left to upper right). An extra parameter $\varepsilon_{\Sigma}=\varepsilon_{M_{1}}+\varepsilon_{M_{2}}$ is introduced for convenience.

\section{The horizontal plane $\left(\varepsilon_{M 1}, \varepsilon_{M 2}, H=c_{0}\right)$}

In Fig. 5 the phase diagram is shown for $H=c_{0}=6$. The first observation is that the phase diagram is dominated by basic surface structures. The phase boundaries of these surface structures can be approximated by a constant $\varepsilon_{\Sigma}$, except for the regions where one of the $\varepsilon_{M}$ is large. With increasing 


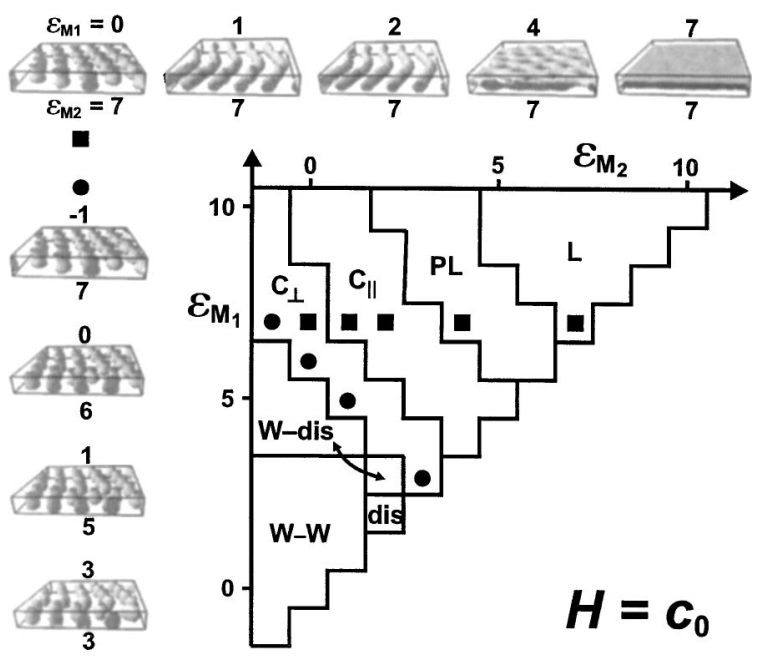

FIG. 5. The phase diagram as a function of $\varepsilon_{M_{1}}$ and $\varepsilon_{M_{2}}$ for $H=c_{0}$. For selected points the microdomain structures are shown. For $\mathbf{Q}, \varepsilon_{\Sigma}=6$. For $\mathbf{\square}$, $\varepsilon_{M_{1}}=7$ and only $\varepsilon_{M_{2}}$ varies

$\varepsilon_{\Sigma}$ we observe the same sequence of structures, $\mathrm{W} \rightarrow$ dis $\rightarrow \mathrm{C}_{\perp} \rightarrow \mathrm{C}_{\|} \rightarrow \mathrm{PL} \rightarrow \mathrm{L}$ as we do for the case of equal interfaces. This is also the case when $\varepsilon_{M_{1}}$ is constant and only $\varepsilon_{M_{2}}$ is varied. In Fig. 5, part of this sequence is shown for $\varepsilon_{M_{1}}=7$.

In the lower left part of the diagram a wetting layer (W) is always present, since the $A$-block is attracted to the surface. Off diagonal we also observe a hybrid W-dis region, which is an intermediate between the region dominated by wetting layers and structures oriented perpendicular to the film plane.

In a previous work ${ }^{9}$ the extend of the effective surface field was determined to be approximately $c_{0}$. The fact that phase boundaries are determined by $\varepsilon_{\Sigma}$ indicates that surface fields are simply additive for this film thickness and phase transitions occur when a certain threshold value is reached.

\section{The horizontal plane $\left(\varepsilon_{M 1}, \varepsilon_{M 2}, H=3 / 2 c_{0}\right)$}

In Fig. 6 the phase diagram is shown for $H=3 / 2 c_{0}=9$. The phase diagram is dominated by hybrid structures, which are combinations of basic surface structures.

In the part of the phase diagram where a wetting layer is present [Fig. 6(b)] the film thickness is effectively reduced by $c_{0} / 2$ grid points. The microdomain structures at the other interface follow with increasing $\varepsilon_{M_{1}}$ the same sequence $\mathrm{C}_{\perp}$ $\rightarrow \mathrm{C}_{\|} \rightarrow \mathrm{PL} \rightarrow \mathrm{L}$ as for $H=c_{0}$ with increasing $\varepsilon_{\Sigma}$.

In the part of the phase diagram where no wetting layer is present, we observe mostly $C_{\perp}$ and $C_{\perp}$ coexisting with $C_{\|}$.

For equal interactions at both interfaces, $\mathrm{C}_{\perp}$ forms, as the film thickness is incommensurable to the natural layer thickness. ${ }^{16}$ The stability of $C_{\perp}$ is limited to a narrow region close to the line with equal interactions at the two interfaces. In other regions where no wetting layer forms, the hybrid structure $\mathrm{C}_{\perp} \mathrm{C}_{\|}$forms and sometimes coexists with $\mathrm{C}_{\|}$. (a)

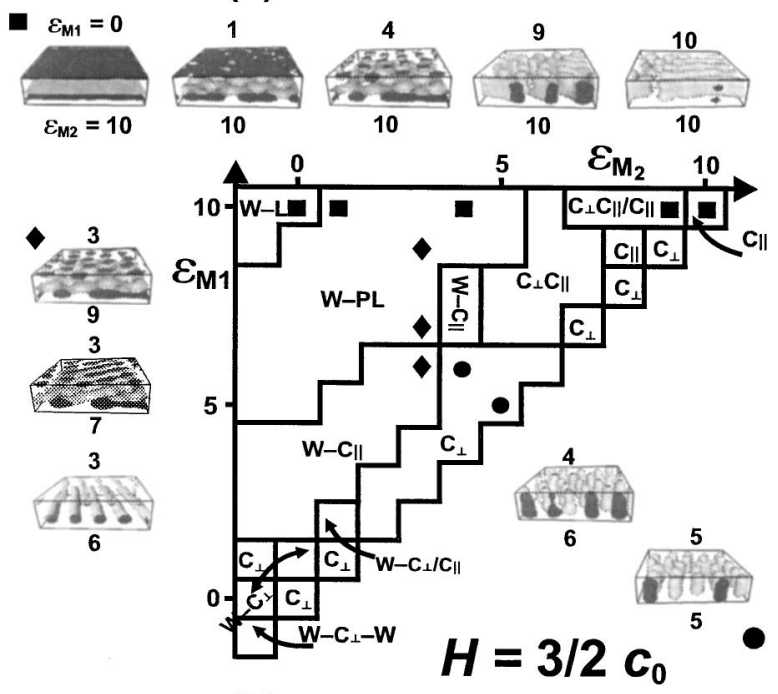

(b)

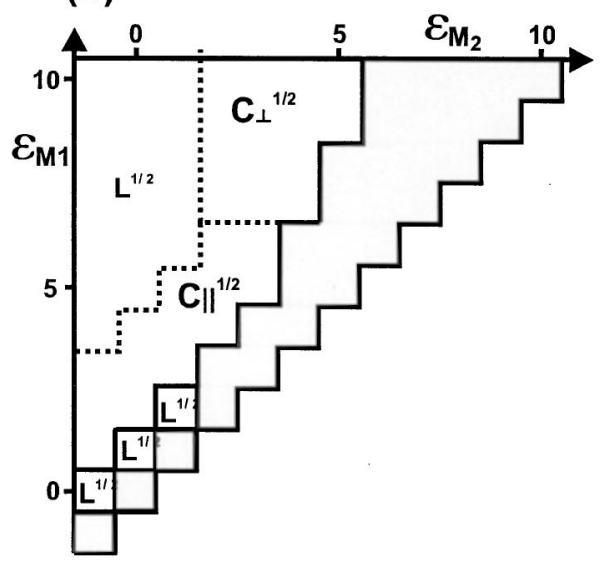

FIG. 6. (a) The phase diagram as a function of $\varepsilon_{M_{1}}$ and $\varepsilon_{M_{2}}$ for $H$ $=3 / 2 c_{0}$. For selected points the microdomain structures are shown. For $\square$, $\varepsilon_{M_{1}}=10$ and only $\varepsilon_{M_{2}}$ varies. For $\bigcirc, \varepsilon_{\Sigma}=10$. Dependence of the structure of the wetting layer on the underlying structure $\left(\bullet, \varepsilon_{M_{2}}=3\right)$. (b) The structure of the wetting layer in (a). The notation is according to the Fig. 4(b).

\section{The horizontal plane $\left(\varepsilon_{M 1}, \varepsilon_{M 2}, H=2 c_{0}\right)$}

In Fig. 7 the phase diagram is shown for $H=2 c_{0}=12$. We observe many different hybrid structures. The surface structures formed at each interface are rather independent of each other and follow with an increase of $\varepsilon_{M_{1}}$ or $\varepsilon_{M_{2}}$ the usual sequence of surface structures $\mathrm{W} \rightarrow \mathrm{C}_{\perp} \rightarrow \mathrm{C}_{\|} \rightarrow \mathrm{PL} \rightarrow \mathrm{L}$ as observed at the surface of thick films. ${ }^{16,17}$

In the center of the phase diagram, for intermediate value of $\varepsilon_{M_{1}}$ and $\varepsilon_{M_{2}}$, we see a tiny region of $\mathrm{C}_{\|} \mathrm{C}_{\perp}$ which connects to a region of $\mathrm{C}_{\perp}$ where $\mathrm{C}_{\perp}$ is stabilized at both interfaces.

For $\varepsilon_{M_{2}} \approx-1$, when only one wetting layer forms we observe $\mathrm{C}_{\|} \mathrm{C}_{\perp}$ and $\mathrm{C}_{\perp} \mathrm{C}_{\|}$. In between two wetting layers, $\mathrm{C}_{\|}$ and $\mathrm{PL}$ can form. In the regions where a wetting layer forms the microdomain structures in the remaining part of the film resemble the phase behavior observed for $H=3 / 2 c_{0}$ and $H$ $=c_{0}$. In case of presence of a wetting layer (for example, $\mathrm{W}-\mathrm{C}_{\|}$) or two or several layers of different structures (for example, $\mathrm{PL}-\mathrm{C}_{\|}$), the thickness where each individual mor- 


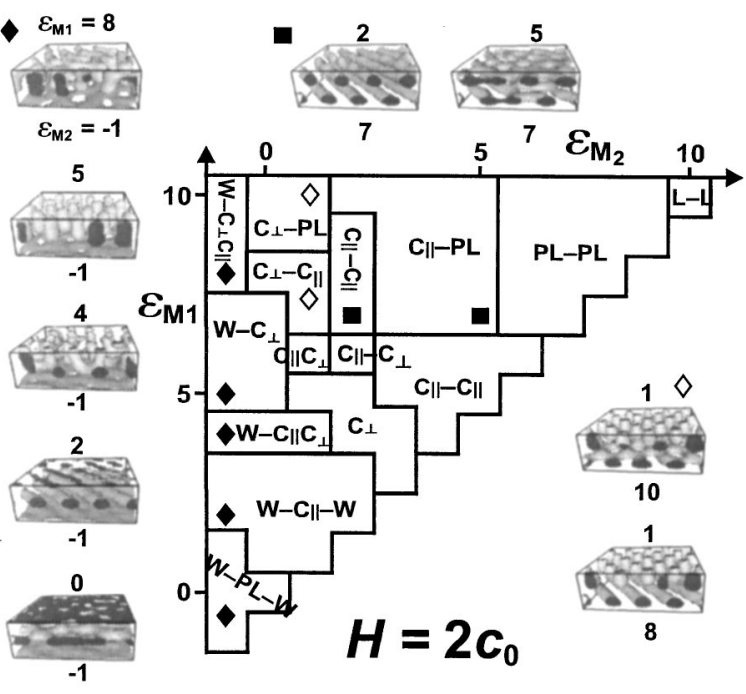

FIG. 7. The phase diagram as a function of $\varepsilon_{M_{1}}$ and $\varepsilon_{M_{2}}$ for $H=2 c_{0}$. For selected points, the microdomain structures are shown. For $\downarrow, \varepsilon_{M_{2}}=-1$; for $\diamond, \varepsilon_{M_{2}}=1$; for $\mathbf{\square}, \varepsilon_{M_{1}}=7$.

phology can be formed $\left(\mathrm{C}_{\|}\right.$in this case) is effectively reduced. This reduced film thickness we call the effective film thickness $H_{\text {eff }}$. In case of the presence of one wetting layer, $H_{\text {eff }}=H-c_{0} / 2$. If two wetting layers are present, $H_{\text {eff }}=H$ $-c_{0}$.

\section{Shape modulations of cylinders}

A feature not represented in the phase diagrams shown in Figs. 4-7 are shape modulations of $\mathrm{C}_{\perp}$ and $\mathrm{C}_{\|}$(Figs. 8 and 9). Shape modulations are an important physical phenomenon as they show how transitions between different basic microdomain structures occur.

1. The $C_{\|}$structure We first discuss shape modulations of $\mathrm{C}_{\|}$. This structure appears to be a flexible structure and adjusts its shape to a rather large range of film thickness. The ideal $\mathrm{a}^{\prime}$-shape is observed when the effective film thickness is commensurable with the natural layer spacing $c_{0}$. This is observed for $H=c_{0}$ [Fig. 8(b)], $H=3 / 2 c_{0}$ (Fig. 9) when a wetting layer forms at one interface $\left(\mathrm{W}-\mathrm{C}_{\|}\right)$, for $H=2 c_{0}$ [Fig. 10(b)] when two wetting layers form $\left(\mathrm{W}-\mathrm{C}_{\|}-\mathrm{W}\right)$ and in the case of $\mathrm{C}_{\|}-\mathrm{C}_{\|}, \mathrm{PL}-\mathrm{C}_{\|}$, and $\mathrm{C}_{\|}-\mathrm{C}_{\perp}$.

When the effective film thickness deviates from the natural layer spacing $c_{0}$ the cylinder shape adjusts. If the thickness $H_{\text {eff }}<c_{0}$ the $\mathrm{C}_{\|}$is compressed in the $z$ direction and when $H_{\text {eff }}>c_{0}$ the $\mathrm{C}_{\|}$is elongated (Fig. 9, shape $\mathrm{c}^{\prime}$ ). The elongated $\mathrm{c}^{\prime}$-shape is observed for equal interactions at the two interfaces, for example, $\varepsilon_{M_{1}}=\varepsilon_{M_{2}}=10$ and $H=3 / 2 c_{0}$ (see Fig. 9). The shape of the cylinder cross section varies depending on the surface field from the neighboring interfaces. In all cases a thin $B$-rich layer is present at the top and bottom of the cylinders. Elongated cylinders are usually stabilized by high surface fields.

In the case of weaker surface fields and commensurable effective film thickness, cylinders can form undulations at
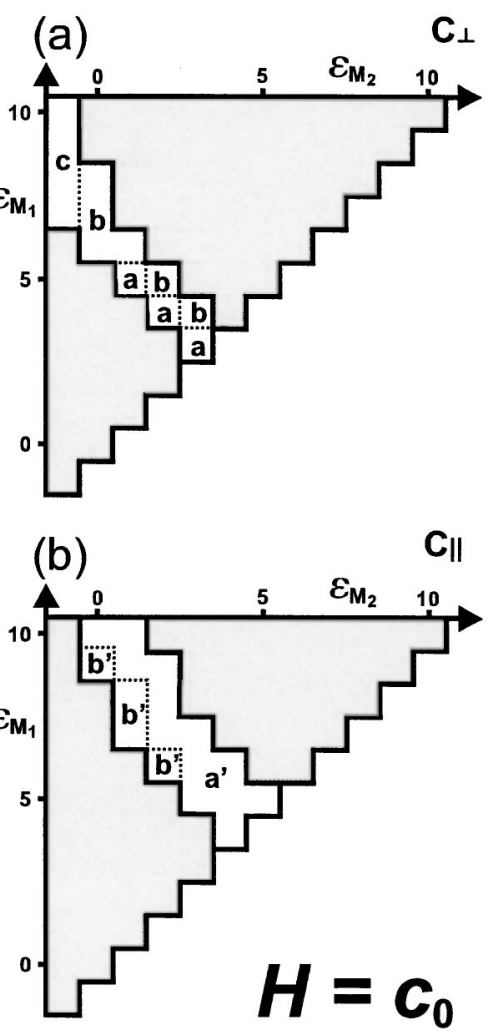

FIG. 8. Shape modulations of $\mathrm{C}_{\perp}$ and $\mathrm{C}_{\|}$for $H=c_{0}$. The complete phase diagram is shown in Fig. 5. Crops of the simulation box showing representative shapes of individual microdomains in different regions of the phase diagram are shown. Different shapes of $\mathrm{C}_{\perp}$ are marked by the letters $\mathrm{a}, \mathrm{b}, \mathrm{c}$, representing: symmetric shape, touching both surfaces (shape a), touching the surface at one side (shape b) and touching the surface at one side and thickened in the center (shape c). Different shapes of $\mathrm{C}_{\|}$are marked by letters $a^{\prime}$ and $b^{\prime}$ representing: The perfect cylinders (shape $a^{\prime}$ ); cylinders modulated in hight from one side (shape $b^{\prime}$ ).

one side of the film (Fig. 8, shape $\mathrm{b}^{\prime}$ ). This shape forms at the boundary between the $\mathrm{C}_{\|}$and $\mathrm{C}_{\perp}$ phases, see, for example, Fig. 8(b). The $\mathrm{b}^{\prime}$-shape can also be considered as cylinders with very short necks.

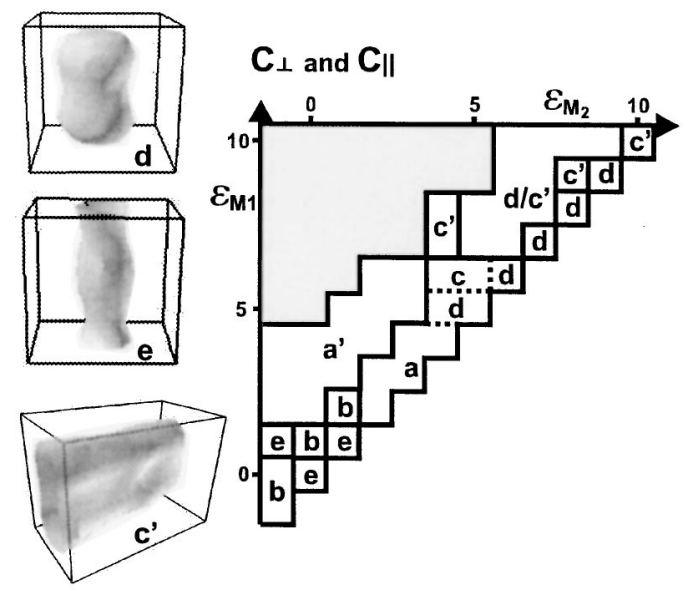

FIG. 9. Shape modulations of $\mathrm{C}_{\perp}$ and $\mathrm{C}_{\|}$for $H=3 / 2 c_{0}$. See Fig. 6 for the complete phase diagram. The classification is according to the scheme shown in Fig. 8. Additional shapes are classified in this figure. For $\mathrm{C}_{\perp}$ : symmetric cylinders not connected to interfaces (shape d) and touching the interface at both sides and thickened in the middle (shape e); for $\mathrm{C}_{\|}$: elongated cylinders (shape $\mathrm{c}^{\prime}$ ). 
2. The $C_{\perp}$ structure We also observe shape modulations of $\mathrm{C}_{\perp}$. The symmetric shape, touching both surfaces [Fig. 8(a), shape a] is only observed in a region around $\varepsilon_{M_{1}}$ $=\varepsilon_{M_{2}} \approx 3$ for $H=c_{0}, 3 / 2 c_{0}$ and $2 c_{0}$. For $H=c_{0}$ this region extends off the diagonal along the line $\varepsilon_{\Sigma}=6$ [Fig. 8(a)]. Next to this region, for dissimilar interfaces, the $\mathrm{C}_{\perp}$ adopts the $b$-shape, touching the surface at one side, and eventually the c-shape, touching the surface at one side and thickened in the center [Fig. 8(a)], when the interaction at the two interfaces are very dissimilar. This sequence of shape transitions occurs for $H=c_{0}$ along the line $\varepsilon_{\Sigma}=6$.

For $H=3 / 2 c_{0}$ different shapes of $\mathrm{C}_{\perp}$ occur along the line $\varepsilon_{M_{1}}=\varepsilon_{M_{2}}$ and extend locally off diagonal. We consider the diagonal for decreasing $\varepsilon_{\Sigma}$. Symmetric cylinders, not connected to the interfaces form (shape d, Fig. 9) for large values of $\varepsilon_{\Sigma}$, which have a dumbbell shape. The d-shape transforms to the a-shape and then transforms to the e-shape for $\varepsilon_{M_{1}}=\varepsilon_{M_{2}}=1$. The e-shape touches the interface at both sides of the film and is a bit thickened in the middle. It forms at $H=3 / 2 c_{0}$ in the absence of wetting layer for small values of $\varepsilon_{M}$ at both interfaces.

For the smallest values of $\varepsilon_{M}$, where $\mathrm{W}-\mathrm{C}_{\perp}-\mathrm{W}$ forms, $\mathrm{C}_{\perp}$ adopts the d-shape. Next to the diagonal, for dissimilar interfaces, $\mathrm{C}_{\perp}$ has c- or d-shapes, depending on the strength of the surface field at the interface next to the end of the cylinder.

In general, when a wetting layer forms, $\mathrm{C}_{\perp}$ never connects to it. $\mathrm{C}_{\perp}$ also never connects to PL and L. $\mathrm{C}_{\perp}$ can, but does not always, connect to $\mathrm{C}_{\|}$, when the two phases form in neighboring layers.

\section{B. General mechanisms of structure formation}

The complex interplay between the strength of the two surface fields, their dissimilarity and the film thickness can be summarized as follows:

(i) For thickness $H=c_{0}$, the phase diagram is dominated by basic surface structures and the film thickness is such that only shape modulations can occur;

(ii) for incommensurable thickness $H=3 / 2 c_{0}$, the phase diagram is dominated by hybrid structures. We observe combinations of wetting layers and basic surface structures as well as perpendicular cylinders, parallel cylinders and coexistence of $\mathrm{C}_{\perp}$ and $\mathrm{C}_{\|}$;

(iii) for thickness $H=2 c_{0}$, the surface structures formed at each surface are rather independent of each other and the microdomain structure depends on how the two surface structures fit and sometimes connect to each other.

An important understanding is in the role of the wetting layer, as it screens the interface-bead interactions and effectively reduces the thickness, from $H=c_{0}$ to $H=1 / 2 c_{0}, H$ $=3 / 2 c_{0}$ to $H=c_{0}$ or $H=1 / 2 c_{0}$ and $H=2 c_{0}$ to $H=3 / 2 c_{0}$ or $H=c_{0}$. This effect is discussed below in Sec. IV B 2. In the following we give an overview of the main mechanisms of structure formation in thin films.

\section{Addition of surface fields}

We found that transitions between surface structures occur when the surface field exceeds a certain threshold value. From previous work we determined that the extend of the surface field is limited to about one microdomain thick region next to the interface. ${ }^{9,10}$ For thin films the surface fields from both interfaces act on the whole film and add. This results in a reduction of the value of $\varepsilon_{\Sigma}$ that is necessary to cause transitions to surface reconstructions in thinner films.

The boundaries of the different phases at $H=c_{0}$ follow approximately the expression $a \leqslant \varepsilon_{\Sigma} \leqslant b$ (with constant $a$ and $b$ ). For the different phases these are: $a=8$ and $b=10$ for $\mathrm{C}_{\|} ; a=11$ and $b=13$ for PL, and $a=13$ for L. Further away from the symmetry line $\varepsilon_{M_{1}}=\varepsilon_{M_{2}}$ the boundaries deviate slightly from this simple approximation.

The interference of surface fields plays a smaller role and the effect is less pronounced in thicker films. For the film thickness $H=2 c_{0}$ the threshold values for transitions between structures are shifted to higher values: $a=8$ and $b$ $=12$ for $\mathrm{C}_{\|} ; a=13$ and $b=19$ for PL, and $a=20$ for L. The threshold values for $\mathrm{C}_{\perp}$ are $a=6, b=7$ for $H=c_{0}$ and $H$ $=2 c_{0}$. They do not change with thickness while the surface fields needed to stabilize surface reconstructions rise dramatically.

\section{The wetting layer: Screening of the surface field}

An important issue is the presence of a wetting layer. When one wetting layer is present, it effectively reduces the film thickness by $c_{0} / 2 \approx 3$ grid points and gives rise for an effective interface for the reduced film (the so-called screening $\operatorname{effect}^{10}$ ). As the wetting layer screens the substrate from the reduced part of the film, the interaction of the effective interface is different, but hard to determine explicitly. A complicating factor is the structure of the wetting layer. For $\varepsilon_{M}$ $\leqslant-2$ (Fig. 4), the wetting layer is homogeneous. For $\varepsilon_{M}$ $\approx 0$ the structure of wetting layer depends on the neighboring film structure.

We found that the thickness of the wetting layer is three grid cells everywhere, which means that we can speak of a half structure. Accordingly, we can consider the homogeneous wetting layer as a half lamella $\left(\mathrm{L}^{1 / 2}\right)$ and the structured wetting layers as a half perforated lamella $\left(\mathrm{PL}^{1 / 2}\right)$, half parallel cylinders $\left(\mathrm{C}_{\|}^{1 / 2}\right)$, and extremely short perpendicular cylinders $\left(C_{\perp}^{1 / 2}\right)$. Although the mean value of the $A$ density in the wetting layer changes significantly with structuring, the thickness of the wetting layer is not affected. A qualitative conclusion is that the effective surface field next to the wetting layer depends on the structure of the wetting layer, which on its turn depends on the strength of the surface field.

The following combinations of thin film structures and structured wetting layers are observed: $\mathrm{L}^{1 / 2}$ next to a $\mathrm{L}, \mathrm{C}_{\perp}^{1 / 2}$, $\mathrm{PL}^{1 / 2}$, and $\mathrm{L}^{1 / 2}$ next to a PL, $\mathrm{C}_{\|}^{1 / 2}$, and $\mathrm{L}^{1 / 2}$ next to a $\mathrm{C}_{\|}$, and $\mathrm{PL}^{1 / 2}$ and $\mathrm{L}^{1 / 2}$ next to a $\mathrm{C}_{\perp}$ structure. The limited number of combinations is due to restrictions caused by the chain architecture.

A good example of the screening effect is the transition of $\mathrm{W}-\mathrm{C}_{\|}-\mathrm{W}$ to $\mathrm{W}-\mathrm{PL}-\mathrm{W}$, found between the two wetting layers, in case the interaction $\varepsilon_{\Sigma}$ is decreased (see $\downarrow$ in Fig. 
7). This transition is in contrast to the transition from $C_{\|}$to $\mathrm{PL}$ in the absence of a wetting layer that takes place with an increase of the surface field. This phenomenon can be explained by the fact that the film thickness is effectively reduced to $H=c_{0}$, and the top wetting layer transforms from a structured one $\left(\mathrm{C}_{\|}^{1 / 2}\right)$ to a homogeneous wetting layer $\left(\mathrm{L}^{1 / 2}\right)$ with decreasing $\varepsilon_{M_{1}}$, such that the surface field is finally fully screened in the middle of the film for the smallest value of $\varepsilon_{M_{1}}$. The effective surface field increases with the wetting layer transforming from $\mathrm{C}_{\|}^{1 / 2}$ to $\mathrm{L}^{1 / 2}$, and reaches a maximum at $\mathrm{W}=\mathrm{L}^{1 / 2}$.

A similar mechanism is observed in the $\mathrm{W}-\mathrm{C}_{\perp} \mathrm{C}_{\|}$ $\rightarrow \mathrm{W}-\mathrm{C}_{\perp} \rightarrow \mathrm{W}-\mathrm{C}_{\|} \mathrm{C}_{\perp}$ transition in the presence of one wetting layer $\left(\diamond\right.$, Fig. 7 ): at $\varepsilon_{M_{1}}=5, \mathrm{C}_{\perp}$ with a symmetric $\mathrm{d}$-shape forms next to the structured wetting layer $\mathrm{PL}^{1 / 2}$. The direction of the necks switches from $\mathrm{W}-\mathrm{C}_{\|} \mathrm{C}_{\perp}$ (with $\mathrm{W}$ $\left.=\mathrm{L}^{1 / 2}\right)$ at $\varepsilon_{M_{1}}=4$ to $\mathrm{W}-\mathrm{C}_{\perp} \mathrm{C}_{\|}$(with $\left.\mathrm{W}=\mathrm{PL}^{1 / 2}\right)$ at $\varepsilon_{M_{1}}=8$. As $C_{\|}$is formed from the side where the surface field has a higher value, this phenomena reflects the changing location of the surface field maximum from one side of the film to the other.

\section{Basic surface structures}

As observed previously $9,10,16,17,19$ basic parallel surface structures are present in films with commensurable (effective) thickness, where one of blocks preferentially wets the surface. For relatively weak surface fields, $\mathrm{C}_{\|}$is formed. With increasing strength of the surface field noncylindrical structures are induced and a decrease of curvature of the $A-B$ interface in the closest layer to the surface is observed: $\mathrm{C}_{\|}$transforms first to PL and then to $\mathrm{L}$ (which has the same symmetry as the surface). With increasing film thickness these phase boundaries shift to higher surface field strength: An effect that can be attributed to interference of surface fields from both surfaces (see Sec. IV B 1).

The basic surface structure $\mathrm{C}_{\perp}$ differs from $\mathrm{C}_{\|}$only in orientation. One source of this structure is a balance between enthalpic and entropic contributions to the free energy: ${ }^{9}$ The weak repulsion of the $A$-component from the surface is balanced by a gain in entropy when the shortest part of the chains is close to the surface. In our case, the interactions at each interface are mostly not equal, and there is a subtle balance. For small thickness, $\mathrm{C}_{\perp}$ is stable further away from the diagonal $\varepsilon_{M_{1}}=\varepsilon_{M_{1}}$ into higher $\varepsilon_{M}$ values $\left(\varepsilon_{\Sigma}=6,7\right)$. For larger commensurable film thickness, the stability region of $\mathrm{C}_{\perp}$ shrinks to a region close to the diagonal (at the same $\varepsilon_{\Sigma}=6,7$ values).

Another source of $\mathrm{C}_{\perp}$ is incommensurability; when the thickness of the film is incommensurable with the natural periodicity of the bulk microdomain structure. In this case the formation of $\mathrm{C}_{\|}$is frustrated and $\mathrm{C}_{\perp}$ often forms throughout the film. However, perfect $\mathrm{C}_{\perp}$ structures are only observed close to the symmetry line $\varepsilon_{M_{1}}=\varepsilon_{M_{2}}$; further away from this line, coexistent parallel and perpendicular structures as well as connected structures are dominant.
TABLE I. All possible combinations of basic and connected structures in two layers. Hybrid structures observed in this work are marked with $\times$; other combinations, which are not classified as hybrids are denoted as $\bigcirc$. The structure $\mathrm{O}^{+}$is out of the range of this article, but was observed for the symmetric case and a larger parameter space (Ref. 16). The structure $X^{*}$ is equal to $\mathrm{C}_{\perp} \mathrm{C}_{\|}$in our notation. Structures with superscript letters were observed in experiments.

\begin{tabular}{lccccccc}
\hline \hline- & $\mathrm{W}$ & $\mathrm{C}_{\perp}$ & $\mathrm{C}_{\|}$ & $\mathrm{PL}$ & $\mathrm{L}$ & $\mathrm{C}_{\perp} \mathrm{C}_{\|}$ & $\mathrm{C}_{\|} \mathrm{C}_{\perp}$ \\
\hline $\mathrm{W}$ & $\bigcirc$ & $\times$ & $\times$ & $\times$ & $\times$ & $\times$ & $\times$ \\
$\mathrm{C}_{\perp}$ & $\times$ & $\bigcirc$ & $\times$ & $\times^{\mathrm{a}}$ & & $\times^{*}, \mathrm{~b}$ & \\
$\mathrm{C}_{\|}$ & $\times$ & $\times$ & $\bigcirc$ & $\times$ & & $\times$ & \\
$\mathrm{PL}$ & $\times$ & $\times^{\mathrm{a}}$ & $\times$ & $\bigcirc$ & & & \\
$\mathrm{L}$ & $\times$ & & & & $\bigcirc^{+}$ & & \\
$\mathrm{C}_{\perp} \mathrm{C}_{\|}$ & $\times$ & $\times^{*}, \mathrm{~b}$ & $\times$ & & & & \\
$\mathrm{C}_{\|} \mathrm{C}_{\perp}$ & $\times$ & & & & & & \\
\hline \hline
\end{tabular}

${ }^{\text {a Harrison et al. (Ref. 8) (spheres cannot be distinguished from the very short }}$ $\mathrm{C}_{\perp}$ ).

${ }^{\mathrm{b}}$ Konrad et al. (Ref. 7).

\section{Hybrid structures}

In the classification of microdomain structures for equal interactions with the interfaces, ${ }^{16}$ hybrid structures were absent. Here, we aim to extend the classification scheme with these new structures. The hybrid structures can be classified in the following three classes:

(1) any combination of not-connected different basic surface structures $\left(\mathrm{W}, \mathrm{C}_{\perp}, \mathrm{C}_{\|}, \mathrm{PL}, \mathrm{L}\right)$. These microdomain structures appear when the surface fields at each of the interfaces supports different surface structures. For large thickness, these combinations are separated by layers of the bulk (C) morphology;

(2) connected basic structures. The only observed connected basic structures are combinations of parallel and perpendicular cylinders: $\mathrm{C}_{\|} \mathrm{C}_{\perp}$ and $\mathrm{C}_{\perp} \mathrm{C}_{\|}$;

(3) combination of (1) and (2). In the explored parameter range, we only observed $\mathrm{W}-\mathrm{C}_{\|} \mathrm{C}_{\perp}, \mathrm{W}-\mathrm{C}_{\perp} \mathrm{C}_{\|}$, and $\mathrm{C}_{\|}-\mathrm{C}_{\perp} \mathrm{C}_{\|}$.

The behavior of the (bulk) cylindrical structures $\left(\mathrm{C}_{\|}, \mathrm{C}_{\perp}\right)$ is somewhat different from the surface reconstructions W, PL, and L. An important observation is that connected $C_{\|} C_{\perp}$ and $C_{\perp} C_{\|}$structures are present at several film thickness due to their flexibility in adopting to different thickness. In experiments, this structures are observed and refered to as "cylinders with necks." 7 The $\mathrm{C}_{\|}$is the only structure $\mathrm{C}_{\perp}$ can connect to when the two structures are simultaneously formed in neighboring layers. The phase boundaries between these two basic structures $\mathrm{C}_{\|}$and $\mathrm{C}_{\perp}$ are less distinct than boundaries between cylindrical and noncylindrical structures, as both $\left(\mathrm{C}_{\|}\right.$and $\left.\mathrm{C}_{\perp}\right)$ are able to modulate their shapes. In particular, $\mathrm{C}_{\|}$transforms with increasing film thickness continuously to $\mathrm{C}_{\|} \mathrm{C}_{\perp}$ which leads us to the conclusion that this phase transition is not of first order.

The following Table I summarizes the observed hybrid structures (marked by $\times$ ). The $\mathrm{W}$-dis structure has only been observed for $H=c_{0}$. We claim that all other combinations not marked by $\times$ may be possible, but are not observed in the studied parameter range. 


\section{Coexistence of microdomain structures}

On the boundaries between basic surface structures, we often find laterally coexisting structures within one layer. These structures are also observed in experiments. ${ }^{16}$ As in experiments, the system can have difficulties to overcome shallow energy barriers in the free energy landscape. The coexisting structures (for example, $\mathrm{PL} / \mathrm{C}_{\|}$) are, therefore, found when the free energy differences between the two structures is relatively small. Due to our dynamic scheme the system can sometimes visit long-living metastable states.

\section{Comparison to other results}

\section{Comparison to confined lamellae forming systems}

As mentioned in the introduction, several groups have studied thin films of lamella forming block copolymers (for a review, see Ref. 3). Here, we compare our new results for cylinder forming triblock copolymers to existing results for lamella forming systems. In both cases the film thickness and the surface field strength determine the phase diagram. The orientational phase transition $\mathrm{L}_{\|} \rightarrow \mathrm{L}_{\perp} \rightarrow \mathrm{L}_{\|}^{35}$ with increasing thickness is analogous to our $\mathrm{C}_{\|} \rightarrow \mathrm{C}_{\perp} \rightarrow \mathrm{C}_{\|}$transition.

In Fasolka et al., ${ }^{28}$ the effect of dissimilar substrates was considered theoretically using self-consistent field theory and compared to their own experiments. Apart from the two basic surface structures $\mathrm{L}_{\|}$and $\mathrm{L}_{\perp}$, a few hybrid structures were observed. For very small thickness $\left(H<\frac{1}{2} L_{0}\right)$, with $L_{0}$ the equilibrium lamella period, and slightly dissimilar conditions they observed a wetting layer with a perpendicular structure connected to it. (HY in their notation); for larger thickness $\left(H>\frac{3}{4} L_{0}\right)$ and one attracting and one repulsive interface, a wetting layer with a disconnected perpendicular structure, separated by a layer of the other component (AHY in their notation) and an anti-symmetric surface parallel lamellae (AFL) were found. Due to the fact that the calculations are two-dimensional, the exact nature (the in plane structure) of the perpendicular structure cannot be determined.

As in Ref. 28 only ultra-thin films were considered ( $H$ $<L_{0}$ ) and the interaction range considered is limited, a comparison with our system is hard to make. From a conceptual point of view, the observed hybrid structures (HY, AHY, and AFL) can be universally classified in terms of our cylindrical system as $\mathrm{C}_{\|} \mathrm{C}_{\perp}(\mathrm{HY}), \mathrm{W}-\mathrm{C}_{\perp}$ (AHY), and $\mathrm{W}-\mathrm{C}_{\|}$(AFL) structures, respectively. If we consider the sequence $\varepsilon_{M_{2}}$ $=-3, \varepsilon_{M_{1}}=6$ with increasing film thickness in Fig. 4, it may be compared to a line through the phase diagram of the lamella forming system for $R \approx-0.5$ (Fig. 8 in Fasolka et $\left.a l .{ }^{28}\right)$. The observed sequence of microdomain structures with increasing $H$ is HL, AHY, and AFL. This is indeed analogous to the sequence $\mathrm{W}-$ dis, $\mathrm{W}-\mathrm{C}_{\perp}$, and $\mathrm{W}-\mathrm{C}_{\|}$observed in Fig. 4 of the present work.

\section{Comparison to simulations with equal interfaces}

In this section we consider the following question: To what extend does dissimilarity of the interfaces alter the main features of the phase diagrams of Knoll et al. ${ }^{16}$ In order to answer this question, we compare the four newly simulated phase diagram slices for dissimilar interfaces to the simulation phase diagram for equal interfaces $\varepsilon_{M_{1}}$ $=\varepsilon_{M_{2}}$ (Fig. 3 in Knoll et al. ${ }^{16}$ ).

The main difference is the presence of hybrid structures. The hybrid $\mathrm{C}_{\|} \mathrm{C}_{\perp}$ structure was only observed as modulated cylinders for equal interfaces at incommensurable film thickness in the wedge-shape geometry (Fig. 1 in Ref. 16). This structure is not very pronounced and might be a long-living transient state between between $\mathrm{C}_{\|}$and $\mathrm{C}_{\perp}$. On the contrary, in the situation of dissimilar interfaces $\mathrm{C}_{\|} \mathrm{C}_{\perp}$ are well pronounced stable structures. We have checked the stability of the $\mathrm{C}_{\|} \mathrm{C}_{\perp}$ in our case of dissimilar interfaces by extending the time range of the simulation, and found them indeed to be stable.

Concentrating first on the phase diagrams for constant film thickness $H\left(c_{0}, 3 / 2 c_{0}, 2 c_{0}\right)$ we observe that for the smallest $H=c_{0}$, changing either $\varepsilon_{M_{1}}$ or $\varepsilon_{M_{2}}$ only leads to a shift of the phase boundaries, apart from shape modulations far away from the diagonal line $\varepsilon_{M_{1}}=\varepsilon_{M_{2}}$. For the incommensurable $H=3 / 2 c_{0}$, a small change in one of the surface interaction values $\varepsilon_{M_{1}}$ or $\varepsilon_{M_{2}}$ leads to a drastic change of the film structure. Away from the diagonal line of equal surface interactions, we observe large regions dominated by wetting layers or hybrid structures $\mathrm{C}_{\perp} \mathrm{C}_{\|}$. In the presence of wetting layers, the transitions of the structures in the remaining film follow the route $\mathrm{C}_{\perp} \rightarrow \mathrm{C}_{\|} \rightarrow \mathrm{PL} \rightarrow \mathrm{L}$ with increasing $\varepsilon_{M_{\alpha}}$. For $H=2 c_{0}$, large structural changes appear only in the region where one $\varepsilon_{M_{\alpha}}$ is small and the other large.

Upon comparing Fig. 3 in Knoll et al. ${ }^{16}$ with the phase diagram shown in Fig. 4 of the present article, where $\varepsilon_{M_{1}}$ $=6$ and $\varepsilon_{M_{2}}$ was varied, we see that the main features are relatively well preserved. In both phase diagrams we observe nested regions of $\mathrm{C}_{\|}, \mathrm{PL}$ (and $\mathrm{L}$ ), both in the presence and absence of wetting layers. In the case of dissimilar interfaces in regions of negative values of $\varepsilon_{M_{2}}$, the wetting layer is only present at one side of the slit, which shifts this region to somewhat lower $H$ values compared to Fig. 3 in Knoll et al. ${ }^{16}$ In Fig. 3 in Knoll et al. ${ }^{16}$ there is a very distinct and fully connected center region of $\mathrm{C}_{\perp}$ around $\varepsilon_{M}=3$, where the enthalpic contribution balances the entropic effect. This region has outliers at incommensurable $H$ due to the frustration of parallel structures, with an extend that is decreasing with increasing $H$. In Fig. 4 of the present article $\left(\varepsilon_{M_{2}}=6\right)$ a region of similar shape can be found at the same or slightly smaller $\varepsilon_{M_{2}}$ position for $H<7$. For higher $H$ values, this region is absent since the balance of energetic and entropic interaction is not strictly satisfied at both interfaces simultaneously.

\section{Comparison with experiments}

In Knoll et al. ${ }^{16,24}$ the phase diagram for polystyreneblock-polybutadien-block-polystyrene was measured as function of the film thickness and polymer concentration. We have shown ${ }^{16,17}$ that the effect of polymer concentration can be modeled by effective interaction parameters that depend on polymer concentration. In Refs. 16 and 17 we used the sequence of phases at the air-film interface to map the inter- 
action parameters to the experimentally controlled parameter polymer concentration $\Phi_{P}$. We found a very good match with the experimental phase diagram for at $\varepsilon_{A B}=6.5$ and $\varepsilon_{M} \sim \Phi_{P}$.

The interactions between poly-styrene (PS)/polybutadiene (PB) and the silicon substrate have not been determined experimentally. A homogeneous $\approx 10 \mathrm{~nm}$ thick $\mathrm{PB}$ layer was found experimentally to cover the air-polymer interface, ${ }^{36}$ indicating a positive $\varepsilon_{M}$ value at the film-air interface. Moreover, recent experiments show that a wetting layer is present at the silicon substrate ${ }^{24}$ indicating a negative $\varepsilon_{M}$ at the film-substrate interface.

In the simulation phase diagram (Fig. 4), the region where the sequence of phases matches the experiments is a rather narrow region around $\varepsilon_{M_{1}}=\varepsilon_{M_{2}}=6$. An interesting observation is that for $\varepsilon_{M_{1}}<-2$ the same sequence of surface structures is observed as in the experiment, however, with a wetting layer at one side of the film. Moreover, in this part of the phase diagram the sequence of phases with increasing $H$ is rather insensitive to changes of $\varepsilon_{M_{2}}$. This observation led us to an attempt to map out the possible interactions parameters $\varepsilon_{M_{1}}$ and $\varepsilon_{M_{2}}$ that are compatible with the experimentally observed sequence of phases.

In the spirit of Refs. 16 and 17, the constraining information is the experimentally observed sequence of phases with increasing height and the experimental evidence for a $A$-rich wetting layer at the film-substrate interface. ${ }^{24}$ For different $H$ we have done simulations for $\left(\varepsilon_{M_{1}}, \varepsilon_{M_{2}}\right)$ $\in[-10 \cdots-1,4 \cdots 10]$ and determined the regions were the surface structure matches the experiment and forms at the film-substrate interface a wetting layer (Fig. 10). The regions where we observe the same sequence of phases as in the experiment, namely, $\mathrm{W}-$ dis, $\mathrm{W}-\mathrm{C}_{\perp}, \mathrm{W}-\mathrm{C}_{\|}, \mathrm{W}-\mathrm{PL}$, $\mathrm{W}-\mathrm{C}_{\|}, \mathrm{W}-\mathrm{C}_{\|} \mathrm{C}_{\perp}$ is limited by $5 \leqslant \varepsilon_{M_{2}} \leqslant 6$ and is broad in the direction of $\varepsilon_{M_{1}}$. We emphasize that in atomic force microscopy only the lateral structure at the air-film interface is observed and that $\mathrm{C}_{\|} \mathrm{C}_{\perp}$ can be distinguished from $\mathrm{C}_{\perp}$ only by the different spacing of microdomains. ${ }^{7}$

This result demonstrates the screening effect of the wetting layer. The wetting layer reduces the effective film thickness by $c_{0} / 2$ and creates for the remaining film almost equal surface interactions at both "effective" interfaces. Remarkably, this region is rather independent of $\varepsilon_{M_{1}}$. This also explains the good agreement between the experiments and the simulations with equal interfaces, despite the dissimilar interfaces in the experiment.

\section{CONCLUSIONS}

In general, the phase behavior of block copolymer films can be understood in terms of a balance between surface fields and commensurability effects (competition between the bulk equilibrium structural periodicity and the film thickness). We have studied the phase behavior of thin films of cylinder forming triblock copolymers between dissimilar interfaces. Although completely "free" (deformable) interfaces are outside the scope of this article, the situation of dissimilar substrates is closer to the experimental reality than the case

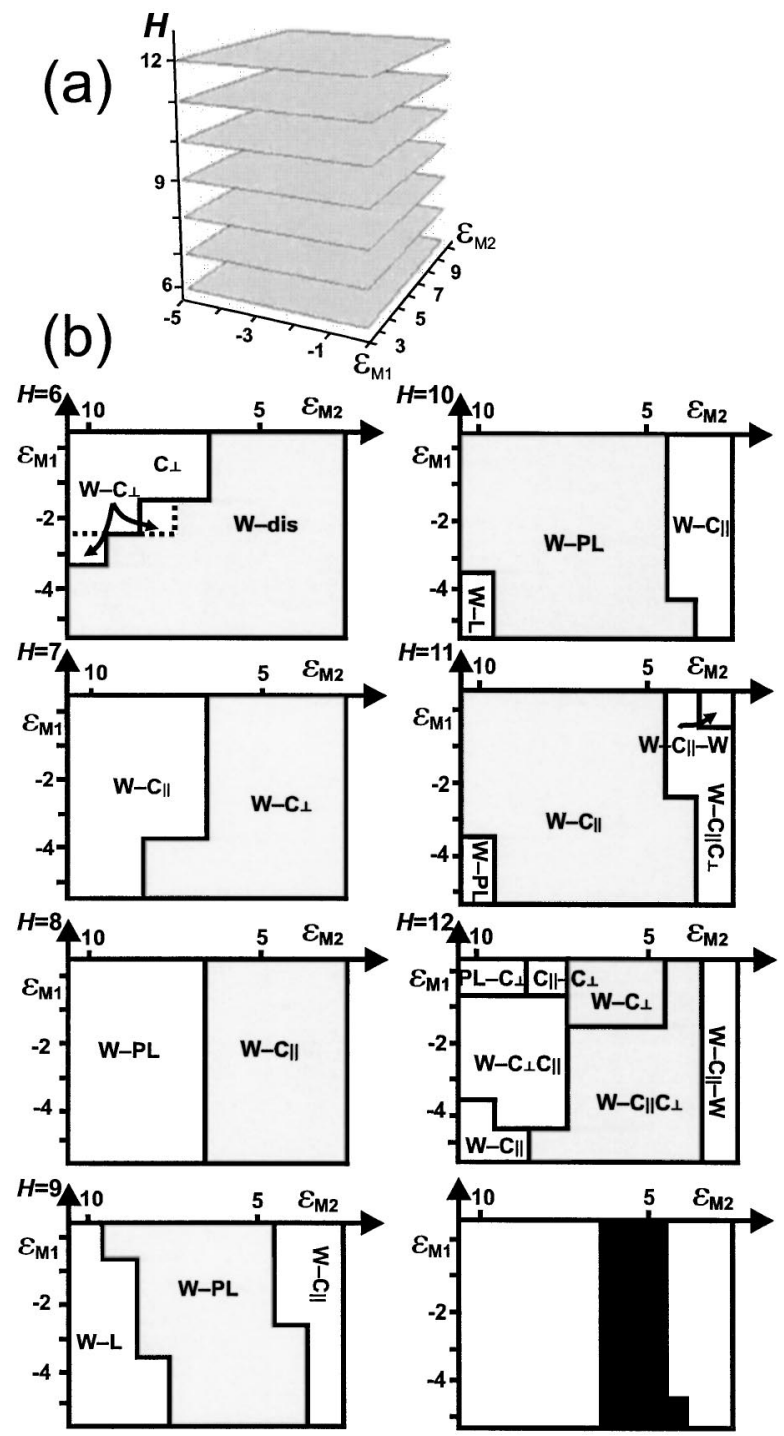

FIG. 10. (a) Position of the planes displayed in (b). (b) Phase diagram for constant film thickness $H=6,7 \ldots 12$. For every $H$, the region where the sequence of surfaces structures at the air-film interface (corresponding to $\varepsilon_{M_{2}}$ ) matches the experiment, is marked in gray. In the bottom right panel the region where for all $H$ the gray regions overlap, is marked in black.

of similar substrates considered before. In many surface supported films, the polymer blocks have different interaction with the supporting substrate and the free surface.

This article is the latest step towards an unifying picture of the phase behavior in thin films of cylinder forming block copolymers. The main feature of systems with dissimilar interfaces is the presence of hybrid structures. These structures are combinations of basic surface structures: $\mathrm{C}_{\|}$(cylinders, oriented parallel to the interface), $\mathrm{C}_{\perp}$ (cylinders, oriented perpendicular to the interface), PL (perforated lamella), $\mathrm{L}$ (lamella), and W (wetting layer). Stable hybrid structures appear when different surface structures are stabilized at each interface of the film. In thick films $\left(H \geqslant 2 c_{0}\right)$ the surface structures at both interfaces form rather independent of each other. In thinner films commensurability effects determine how different structures connect to each other. In addition, additivity of surface fields plays an important role in thin films $\left(H \leqslant 2 c_{0}\right)$. The only connected basic structures 
found are combinations of the bulk microdomain structures $\mathrm{C}_{\|}$and $\mathrm{C}_{\perp}$.

Compared with lamella forming system, ${ }^{3}$ the situation for cylinder forming block copolymers is similar, but much more complex as more combinations of surface structures and reconstructions are involved. By comparing our results to previous work with equal interfaces ${ }^{16,17}$ we have shown that the apparent paradox - the experimentally studied SBS triblock copolymer thin films with dissimilar interfaces can be modeled in great detail with simulations with equal interfaces - is due to the effect of screening of the surface field by a wetting layer.

We propose a general classification of hybrid structures and qualitatively determine the main factors in the formation of these structures. We found that the general mechanisms of structure formation for cylinder forming systems are essentially transposable to the case of lamellar forming system.

The experimental observation of hybrid structures is difficult, as layer by layer imaging of the polymer film with high resolution is required. Techniques capable of doing this are rather new, for instance nanotomography, and still under development. Examples can be found in recent papers. ${ }^{7,29,37}$ In these experiments hybrid structures were found, in agreement with our findings.

Our simulation method allows the in-silico prediction of structure formation in a wide parameter range with remarkable accuracy. The results could be valuable in many nanotechnological applications.

\section{ACKNOWLEDGMENTS}

We thank the NWO-DFG bilateral program, the Deutsche Forschungsgemeinschaft (SFB 481) and the Volkswagenstiftung for financial support. We acknowledge the Stichting Nationale Computer Faciliteiten for providing supercomputer time.

${ }^{1}$ F. Bates and G. Fredrickson, Phys. Today 52(2), 32 (1999).

${ }^{2}$ M. W. Matsen, Curr. Opin. Colloid Interface Sci. 3, 40 (1998).

${ }^{3}$ M. J. Fasolka and A. M. Mayes, Annu. Rev. Mater. Res. 31, 323 (2001).

${ }^{4}$ K. Binder, Adv. Polym. Sci. 138, 1 (2001).

${ }^{5}$ A. Karim, N. Singh, M. Sikka, F. S. Bates, W. D. Dozier, and G. P. Felcher, J. Chem. Phys. 100, 1620 (1994).

${ }^{6}$ L. H. Radzilowski, B. L. Carvalho, and E. L. Rhomas, J. Polym. Sci., Part B: Polym. Phys. 34, 3081 (1996).

${ }^{7}$ M. Konrad, A. Knoll, R. Magerle, and G. Krausch, Macromolecules 33, 5518 (2000).
${ }^{8}$ C. Harrison, M. Park, P. M. Chaikin, R. A. Register, D. H. Adamson, and N. Yao, Macromolecules 31, 2185 (1998).

${ }^{9}$ H. P. Huinink, J. C. M. Brokken-Zijp, M. A. van Dijk, and G. J. A. Sevink, J. Chem. Phys. 112, 2452 (2000).

${ }^{10}$ H. P. Huinink, M. A. van Dijk, J. C. M. Brokken-Zijp, and G. J. A. Sevink, Macromolecules 34, 5325 (2001).

${ }^{11}$ Q. Wang, P. F. Nealey, and J. J. de Pablo, Macromolecules 34, 3458 (2001).

${ }^{12}$ S. Turner, M. Rubenstein, and C. M. Marques, Macromolecules 27, 4986 (1994).

${ }^{13}$ G. Brown and A. Chakrabati, J. Chem. Phys. 101, 3310 (1994); 102, 1440 (1995).

${ }^{14}$ G. G. Pereira, Phys. Rev. E 63, 061809 (2001).

${ }^{15}$ J. Feng, H. Liu, and Y. Hu, Macromol. Theory Simul. 11, 556 (2002); J. Feng and E. Ruckenstein, Polymer 43, 5775 (2002).

${ }^{16}$ A. Knoll, A. Horvat, K. S. Lyakhova, G. Krausch, G. J. A. Sevink, A. V. Zvelindovsky, and R. Magerle, Phys. Rev. Lett. 89, 035501 (2002).

${ }^{17}$ A. Horvat, K. S. Lyakhova, G. J. A. Sevink, A. V. Zvelindovsky, and R. Magerle, J. Chem. Phys. 120, 1117 (2004).

${ }^{18}$ G. Szamel and M. Mueller, J. Chem. Phys. 118, 905 (2003).

${ }^{19}$ G. J. A. Sevink, J. G. E. M. Fraaije, and H. P. Huinink, Macromolecules 35, 1848 (2002).

${ }^{20}$ N. Rehse, A. Knoll, M. Konrad, R. Magerle, and G. Krausch, Phys. Rev. Lett. 87, 5505 (2001); N. Rehse, A. Knoll, R. Magerle, and G. Krausch, Macromolecules 36, 3261 (2003).

${ }^{21}$ C. Auschra and R. Stadler, Macromolecules 26, 6364 (1993).

${ }^{22}$ L. Li, L. Chen, P. Bruin, and M. A. Winnik, J. Polym. Sci., Part B: Polym. Phys. 35, 979 (1997).

${ }^{23}$ H. Stutz, W. Heckmann, P. Pötschke, and K. Wallheinke, J. Appl. Polym. Sci. 83, 2901 (2002).

${ }^{24}$ A. Knoll, R. Magerle, and G. Kraush, J. Chem. Phys. 120, 1105 (2004).

${ }^{25}$ M. Matsen, J. Chem. Phys. 106, 7781 (1997).

${ }^{26}$ D. G. Walton, G. J. Kelogg, A. M. Mayes, P. Lamboy, and T. P. Russel, Macromolecules 27, 6525 (1994).

${ }^{27}$ E. Huang, P. Mansky, T. P. Russel, C. Harrison, P. M. Chaikin, R. A. Register, C. J. Hawker, and J. Mays, Macromolecules 33, 80 (2000).

${ }^{28}$ M. J. Fasolka, P. Benerjee, A. M. Mayes, G. Pickett, and A. C. Balazs, Macromolecules 33, 5702 (2000).

${ }^{29}$ C. Harrison, M. Park, P. M. Chaikin, R. A. Register, D. H. Adamson, and N. Yao, Polymer 39, 2733 (1998).

${ }^{30}$ J. G. E. M. Fraaije, B. A. C. van Vlimmeren, N. M. Maurits, M. Postma, O. A. Evers, C. Hoffmann, P. Altevogt, and G. Goldbeck-Wood, J. Chem. Phys. 106, 4260 (1997).

${ }^{31}$ N. M. Maurits, B. A. C. van Vlimmeren, and J. G. E. M. Fraaije, Phys. Rev. E 56, 816 (1997).

${ }^{32}$ B. A. C. van Vlimmeren, N. M. Maurits, A. V. Zvelindovsky, G. J. A. Sevink, and J. G. E. M. Fraaije, Macromolecules 32, 646 (1999).

${ }^{33}$ N. M. Maurits and J. G. E. M. Fraaije, J. Chem. Phys. 15, 5879 (1997).

${ }^{34}$ G. J. A. Sevink, A. V. Zvelindovsky, B. A. C. van Vlimmeren, N. M. Maurits, and J. G. E. M. Fraaije, J. Chem. Phys. 110, 2250 (1999).

${ }^{35}$ B. L. Carvalho and E. L. Thomas, Phys. Rev. Lett. 73, 3321 (1994).

${ }^{36}$ A. Knoll, R. Magerle, and G. Krausch, Macromolecules 34, 4159 (2001).

${ }^{37}$ R. Magerle, Phys. Rev. Lett. 85, 2749 (2000). 